\title{
ELUCIDACIÓN \\ DE LA FÓRMULA ESTEQUIOMÉTRICA DE NANOHIDROTALCITAS DE Mg Y Ni SINTETIZADAS POR EL MÉTODO DE COPRECIPITACIÓN
}

\author{
ELUCIDATION OF THE STECHIOMETRIC \\ FORMULA OF NANOHYDROTHALCITES OF Mg Y Ni \\ SYNTHESIZED BY THE METHOD OF CO-PRECIPITATION
}

\section{Augusto Rodríguez C. ${ }^{* *}$, Lenys Fernández M. ${ }^{1 *}$, José Domínguez V. ${ }^{2}{ }^{+}$, Gema González V. ${ }^{3,5}$, Omar Martínez M. ${ }^{6}$ \& Patricio Espinoza-Montero ${ }^{1}$}

Recibido: 26 de abril 2021 / Aceptado: 1 de julio 2021

DOI 10.26807/ia.v9i2.212

Palabras clave: Arcillas, Hidrotalcitas, Hidróxidos dobles laminares,

Relación estequiométrica, Síntesis.

Keywords: Clays, Hydrotalcites, Lamellar double hydroxides,

Stoichiometric ratio, Synthesis.

1 Pontificia Universidad Católica del Ecuador, Escuela de Ciencias Químicas, Quito, Ecuador. ("correspondencia: Imfernandez@puce.edu.ec, pespinoza646@puce.edu.ec)

2 Universidad Simón Bolívar, Departamento de Química, Caracas, Venezuela (jrdomin@usb.ve)

3 Yachay Tech University, School of Physical Sciences and Nanotechnology, Urcuqui, Ecuador. (ggonzalez@yachaytech.edu.ec)

4 Grupo CAE INIFTA (Instituto de Investigaciones Fisicoquímicas Teóricas y Aplicadas), La Plata, Argentina. (*correspondencia: augustorodriguez@inifta.unlp.edu.ar)

5 Instituto Venezolano de Investigaciones Científicas, Centro de Ingeniería Materiales y Nanotecnología, Caracas, Venezuela. (gemagonz@gmail.com )

6 Universidad Técnica de Machala, Facultad de Ciencias Químicas y de la Salud, Machala, Ecuador. (emartinez@utmachala.edu.ec) 


\section{RESUMEN}

Los hidróxidos dobles laminares ( $\mathrm{LDH}$, por sus siglas en inglés), también denominados como Hidrotalcitas (HTs), son una clase de materiales arcillosos bidimensionales conocidos por su estructura laminar única, composición versátil y propiedades de intercambio iónico. En este trabajo se reporta la determinación de la relación estequiométrica de arcillas tipo HTs sintetizadas en el laboratorio por el método de co-precipitación. El contenido de metales di y tri-valente que conforman las estructuras de las arcillas fue cuantificado por espectroscopia de absorción atómica por llama. Se sintetizaron dos tipos de HTs, $\mathrm{Ni}(\mathrm{HT}-\mathrm{Ni})$ y Mg (HT-Mg); donde de acuerdo con los cálculos realizados la formula estequiométrica para la primera arcilla es $\mathrm{Ni}_{0,82} \mathrm{Al}_{0,18}(\mathrm{OH})_{2}\left(\mathrm{NO}_{3}\right)_{0,18}$ y $\mathrm{Mg}_{0,80} \mathrm{Al}_{0,20}(\mathrm{OH})_{2}\left(\mathrm{NO}_{3}\right)_{0,20}$ para la segunda, resultados que concuerdan perfectamente con la literatura. Las arcillas sintetizadas fueron caracterizadas por difracción de rayos X, espectroscopia FT-IR y microscopia electrónica de barrido. Los patrones de difracción de rayos $X$ confirmaron la formación de HTs de tamaño de partícula nanométrico, donde la HT-Mg sintetizada posee mejores propiedades cristalinas.

\section{ABSTRACT}

The lamellar double hydroxides (LDH), also known as Hydrotalcites (HTs), correspond to a family of two-dimensional clay materials notorious for their unique lamellar structures, versatile composition and ion exchange properties. In this research, the determination of the stoichiometry ratio of a set of Hydrotalcites clays, synthesized in the lab by the co-precipitated method, is reported. The content from the trivalent and divalent metals that conform the clays structures was determined through flame atomic absorption spectroscopy. Two types of HTs were synthesized, $\mathrm{Ni}(\mathrm{HT}-\mathrm{Ni})$ and $\mathrm{Mg}(\mathrm{HT}-\mathrm{Mg})$; where, according to the resulting calculations, the stoichiometric formulas correspond to $\mathrm{Ni}_{0.82} \mathrm{Al}_{0.18}(\mathrm{OH})_{2}\left(\mathrm{NO}_{3}\right)_{0.18}$ and $\mathrm{Mg}_{0.80} \mathrm{Al}_{0.20}(\mathrm{OH})_{2}\left(\mathrm{NO}_{3}\right)_{0.20}$ respectively, formulas that show great similarities with reported values on the available literature. Furthermore, the synthesized clays were also characterized by X-Ray Diffrac- 
tion, FT-IR spectroscopy and scanning electron microscopy; where the X-Ray diffraction patterns confirmed the formation of nanometric size HTs particles, with the HT-Mg clay showing better crystalline properties.

\section{INTRODUCCIÓN}

Los hidróxidos dobles laminares (LDH, por sus siglas en inglés), denominados en la mayoría de los casos como Hidrotalcitas (HTs), son materiales arcillosos bidimensionales (2D) de composición versátil y propiedades de intercambio aniónico, conocidos por su estructura doble laminar única; esta última constituida por aniones entre capas intermedias y capas huésped con carga positiva (octaédrico $\mathrm{M}(\mathrm{OH})_{6}$, similar a la brucita natural), Figura 1 (Martínez \& Carvajal, 2012).

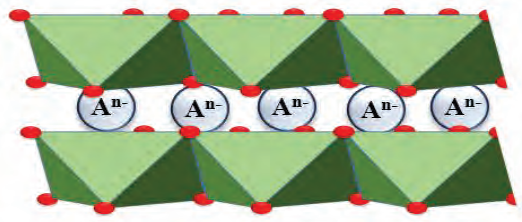

(An-) Aniones

- Grupo ОН

Octaedros $\mathrm{M}(\mathrm{OH})_{6} \mathrm{M}^{\mathrm{II}} / \mathrm{M}^{\mathrm{II}}$

Figura 1. Esquema estructural de los materiales tipo hidrotalcitas
Estos materiales no se encuentran en abundancia en la naturaleza, como las arcillas catiónicas, sin embargo, son fáciles de preparar en el laboratorio de manera sintética y a bajo costo.

Los LDH poseen una composición química general $\left[\mathrm{M}^{2+}{ }_{1-\mathrm{x}} \cdot \mathrm{M}^{3+}{ }_{\mathrm{x}} \cdot(\mathrm{OH})_{2}\right]$ $\left(\mathrm{A}^{\mathrm{n}-}\right)_{\mathrm{x} / \mathrm{n}} \cdot \mathrm{y} \cdot \mathrm{H}_{2} \mathrm{O}$, la cual se obtiene cuando parte del metal divalente, $\mathrm{M}^{2+}$, en el $\mathrm{M}(\mathrm{OH})_{2}$ se reemplaza parcialmente con un trivalente, $\mathrm{M}^{3+}$, de radio iónico similar, donde " $\mathrm{x}$ " generalmente varía de 0,20<x<0,33 (Zhitova et al., 2020; Mishra et al., 2018). Se han descrito otras formulaciones, como las fases ricas en aluminio (Britto, \& Kamath, 2014; Hawthorne \& Cooper, 2013), donde los sitios octaédricos vacíos en el $\mathrm{Al}(\mathrm{OH})_{3}$ (gibbsita, bayerita) están ocupados por cationes de $\mathrm{Li}^{+} \mathrm{u}$ otros cationes metálicos divalentes. Esta inserción crea un exceso de carga po- 
sitiva en las capas, que son compensadas por la intercalación de aniones entre ellas. En la fase Li/Al (Li $\left.\left(\mathrm{Al}(\mathrm{OH})_{3}\right)_{2} \cdot\left(\mathrm{A}^{\mathrm{n}-}\right)_{1 / \mathrm{n} \cdot \mathrm{y}} \mathrm{H}_{2} \mathrm{O}\right)$, solo los cationes $\mathrm{Li}^{+}$ocupan los sitios octaédricos vacíos y diferentes tipos de aniones pueden intercalarse entre las capas. En consecuencia, los LDH ofrecen una oportunidad notable para sintetizar nuevos materiales a través de la intercalación de compuestos entre sus capas, mejorando así sus propiedades químicas por efectos sinérgicos. Los LDH, se han empleado en varios procesos tecnológicos (Stamate et al., 2020) que van desde la producción de energía renovable hasta la purificación y remediación de agua, materiales funcionalizados para nanogeneradores piezoeléctricos y detección de gases, aplicaciones biomédicas, síntesis de nanocompuestos inteligentes híbridos, que involucran tecnologías en expansión como la liberación de medicamentos y el envasado de alimentos. La composición variable y propiedades intrínsecas de los LDH son las principales razones por las cuales su uso catalítico es su principal campo de aplicación, donde exhiben actividades y selectividades importantes en una amplia gama de reac- ciones que se llevan a cabo comúnmente en la industria, que incluyen condensación, alquilación, ciclación, isomerización, etc.

Se han reportado síntesis de LDH por métodos directos e indirectos (Pizzoferrato \& Richetta, 2020), como la coprecipitación, (Zaghloul et al., 2021; Wen et al., 2021) crecimiento hidrotermal, (Hu et al., 2021) síntesis sol-gel, (Pérez et al., 2020) química blanda, (Liu et al., 2021) síntesis electroquímica, (Li et al., 2021; Pan et al., 2021) intercambio aniónico, (Yao et al., 2021; Jeung et al., 2021) y aqueIlos en los que los LDH se utilizan como precursores (Li et al., 2013; Rybka et al., 2021). Las propiedades específicas de los LDH, como la porosidad y el área superficial, se pueden controlar variando el método y las condiciones de síntesis, lo que da lugar a una amplia gama de materiales adaptados a las aplicaciones específicas mencionadas en el párrafo anterior. La estructura de los LDH, también se puede modificar cambiando la combinación de los cationes y los aniones de las capas intermedias para conferirles propiedades específicas (Chaillot et al., 2020; Zheng et al., 2019; Wu et al., 2019). 
En ese sentido, el reto es lograr procesos de síntesis rápidos y ecológicos, los cuales combinados con la alta capacidad de sorción y combinación de los materiales tipo LDH, permitan lograr derivados útiles en diversas aplicaciones, como la adsorción de diversas moléculas, contaminantes orgánicos e inorgánicos, o procesos catalíticos gracias a la generación de sitios básicos activos en la superficie de estos materiales.

La co-precipitación es el método más común para sintetizar LDH, el cual consiste en disolver sales inorgánicas precursoras de LHD en un medio alcalino, a pH constante o en aumento, nunca en disminución. Este método permite controlar la morfología y el tamaño de partícula, dependiendo de la sobresaturación de la solución y del estado de cristalización deseado (Chaillot et al., 2020). Los LHD obtenidos por co-precipitación a baja sobresaturación presentan una cristalinidad superior a los obtenidos en condiciones de alta sobresaturación. Por otro lado, agregar la solución que contiene las sales precursoras en una solución con un pequeño exceso de iones bicarbonatos, puede conducir a la formación de materiales menos cristalinos que el método por baja sobresaturación, pero da lugar a un gran número de pequeños agregados, medianamente nanométricos, debido al efecto de confinamiento entre capas de los iones carbonatos (Solovov et al., 2018). La síntesis de LDH por co-precipitación, puede considerarse un método eficaz para la obtención de materiales cristalinos, particularmente gracias al largo tiempo de duración de la síntesis, el control del $\mathrm{pH}$ y la posibilidad de acoplarle tratamientos hidrotérmicos (Kannan et al., 2004). Sin embargo, debido a las dimensiones micro de las partículas obtenidas por este método, las superficies específicas son menores que las obtenidas por otro tipo de síntesis. Lo anterior constituye un reto importante en la literatura, donde se ha reportado que el uso de cationes metálicos como $\mathrm{Fe}, \mathrm{Zn}, \mathrm{Cu}$ y Ni, permite la posibilidad de modificar las dimensiones de los cristales e influir en la estabilidad térmica de los LDH (Gevers et al., 2019).

Las HTs son LDH particulares, caracterizadas por una relación molar Mg:Al de 3:1, donde el Mg está parcialmente sustituido por Al. En este trabajo, se presenta la determinación 
de las relaciones estequiométricas de nanohidrotalcitas de Mg y Ni sintetizadas por el método de co-precipitación adaptado a una titulación de doble precipitación en solución básica y en presencia de iones carbonatos y nitratos, lo que dio lugar a la obtención de HTs de tamaño nanométrico. Las oportunidades que ofrecen las propiedades únicas de las
HTs de tamaño nanométrico son sumamente amplias (Shekoohi et al., 2017; Manivannan et al., 2013; Dahdah et al., 2020; Bahramian, 2020), y sus aplicaciones están en constante crecimiento, lo que hace extremadamente interesantes investigar nuevas técnicas de síntesis para la obtención de HTs y sus derivados.

\section{MATERIALES Y MÉTODOS}

\section{Reactivos químicos}

Nitrato de aluminio nonahidratado, $\mathrm{Al}\left(\mathrm{NO}_{3}\right)_{3} \cdot 9 \mathrm{H}_{2} \mathrm{O}, 98,5 \%$, Scharlau Chemie; Nitrato de níquel (II) hexahidratado, $\mathrm{Ni}\left(\mathrm{NO}_{3}\right)_{2} \cdot 6 \mathrm{H}_{2} \mathrm{O}, 99 \%$, Merck; Nitrato de magnesio hexahidratado, $\mathrm{Mg}\left(\mathrm{NO}_{3}\right)_{2} \cdot 6 \mathrm{H}_{2} \mathrm{O}, 98,9 \%$, Fisher Scientific; Hidróxido de sodio, $\mathrm{NaOH}, 98$ \%, Eka Nobel.

\section{Equipos}

Difractómetro de rayos $\mathrm{X}$, marca BRUKER, modelo AXS D8, radiación Cu ka; microscopio electrónico de Barrido JEOL JSM-6390 Scanning Electron Microscope; espectrómetro FT-IR Brucker TENSOR"27 y espectrómetro de absorción atómica Per- kin- Elmer modelo 2380 y el análisis termogravimétrico (TGA) se llevó a cabo en una termobalanza Setaram.

\section{Síntesis de Hidrotalcita}

Para la síntesis de las arcillas, se empleó el método de co-precipitación reportado por Miyata, 1983. Se prepararon dos soluciones contentivas, una de 0,25 moles de $\mathrm{Al}\left(\mathrm{NO}_{3}\right)_{3}+$ 0,75 moles de $\mathrm{Ni}\left(\mathrm{NO}_{3}\right)_{2}$, y la otra con 0,25 moles de $\mathrm{Al}\left(\mathrm{NO}_{3}\right)_{3}+0,75$ moles de $\mathrm{Mg}\left(\mathrm{NO}_{3}\right)_{2}$. El contenido de $\mathrm{CO}_{2}$ en ambas soluciones fue eliminado por burbujeo con $\mathrm{N}_{2}$, de alta pureza, por 20 minutos. Posteriormente, cada solución fue titulada con $\mathrm{NaOH} 1 \mathrm{~mol} \mathrm{~L}^{-1}$, gota a gota, hasta lograr la precipitación de las arcillas. 
Los precipitados obtenidos de cada titulación constituyen las arcillas HT de $\mathrm{Mg}-\mathrm{Al}-\mathrm{NO}_{3}(\mathrm{HT}-\mathrm{Mg})$ y de $\mathrm{Ni}-\mathrm{Al}-$ $\mathrm{NO}_{3}(\mathrm{HT}-\mathrm{Ni})$. Los precipitados se filtraron en un crisol de vidrio sinterizado, lavándolos con agua destilada para luego colocarlos en una estufa a $80{ }^{\circ} \mathrm{C}$ por 24 horas. Las HTs sintetizadas se caracterizaron por difracción de rayos $X$, espectroscopia FT-IR, TGA y microscopía electrónica de barrido (SEM, por sus siglas en inglés). El contenido de metales en cada arcilla se determinó mediante espectroscopia de absorción atómica. Previamente a cada caracterización, las HTs se almacenaron en viales dentro de un desecador. Antes de la caracterización, debido a que las arcillas HTs son materiales higroscópicos, una porción de estas fueron deshidratadas en una estufa a $80{ }^{\circ} \mathrm{C}$ 20 minutos. Para los espectros FT-IR, antes de que las HT se enfriaran por completo, se homogeneizaron en $\mathrm{KBr}$ para obtener las mezclas con las cuales se elaboraron las pastillas a ser colocadas en el equipo de espectrofotometría. Para obtener los difractogramas respectivos de cada material, los mismos se trituraron hasta obtenerse un polvo muy fino y homogé- neo que se dispersó sobre el portamuestra del difractómetro formando una capa lo más uniforme posible. Se abarcó un barrido de $3^{\circ}$ hasta $60^{\circ} 2 \theta$.

\section{Determinación del contenido de Al, Ni y Mg mediante espectroscopia de absorción atómica a la llama}

Para este procedimiento, $100 \mathrm{mg}$ de cada arcilla se disolvieron por separado, con 5 gotas de ácido nítrico concentrado llevando luego a un aforo de $100 \mathrm{~mL}$. En ambas soluciones se utilizó $\mathrm{KNO}_{3}$ como supresor de ionización de $\mathrm{Al}$, a una concentración final de $2000 \mathrm{mg} \mathrm{L}^{-1}$ de K. Se utilizó $\mathrm{Sr}\left(\mathrm{SrCl}_{2}\right)$ como agente liberador del Mg a una concentración en solución de $3000 \mathrm{mg} \mathrm{L}^{-1}$. Se construyeron curvas de calibración por espectroscopia de absorción atómica por Ilama, empleando patrones estándar de $\mathrm{Ni}, \mathrm{Al}$ y $\mathrm{Mg}$ en rangos de concentraciones de 2 a $8 \mathrm{mg} \mathrm{L}^{-1}$, de 25 a $110 \mathrm{mg} \mathrm{L}^{-1}$ y de 0,1 a $1 \mathrm{mg} \mathrm{L}^{-1}$, respectivamente. Los patrones fueron preparados conteniendo las mismas concentraciones del $\mathrm{K}$ y del Sr como supresor de ionización y agente liberador, respectivamente. 


\section{RESULTADOS}

Los patrones de difracción de rayos $X$ para las HTs sintetizadas, se muestran en las Figuras $2 \mathrm{a}$ y $2 \mathrm{~b}$, donde se presentan patrones característicos de los hidróxidos dobles laminares y los es- pectros IR obtenidos (Figura 3a y 3b) muestran bandas de transmitancia reportadas en anterioridad para este tipo de arcillas (Wiyantoko et al., 2015)
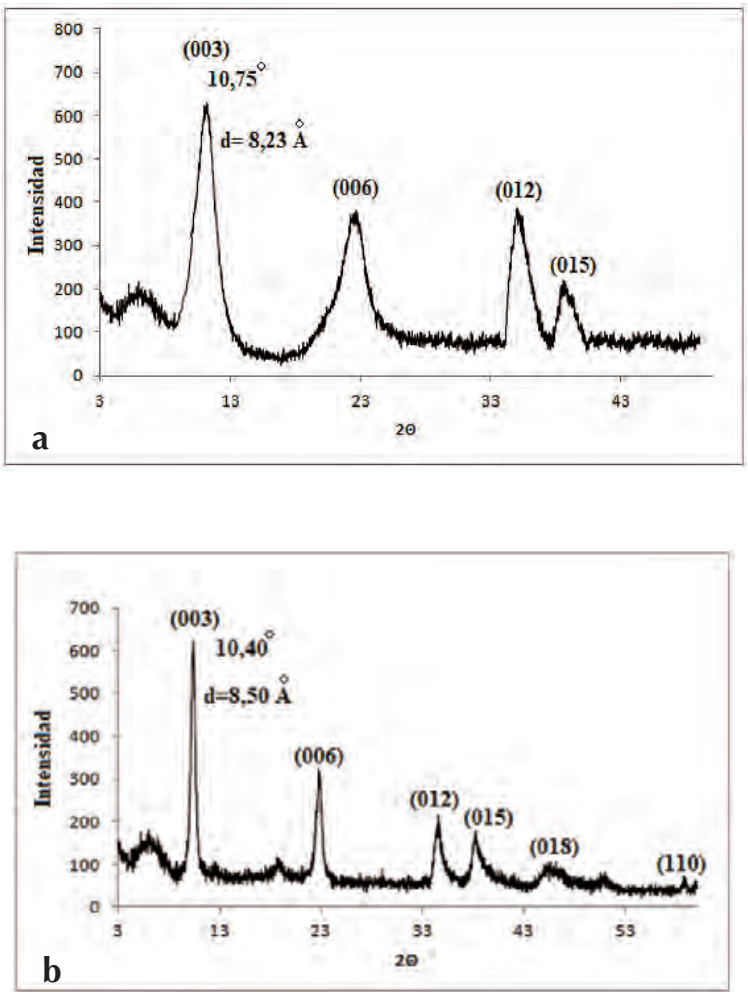

Figura 2. Patrón de difracción de rayos $\mathrm{X}$ obtenido para:

a) la arcilla $\mathrm{HT}-\mathrm{Ni}$, b) la arcilla $\mathrm{HT}-\mathrm{Mg}$ 

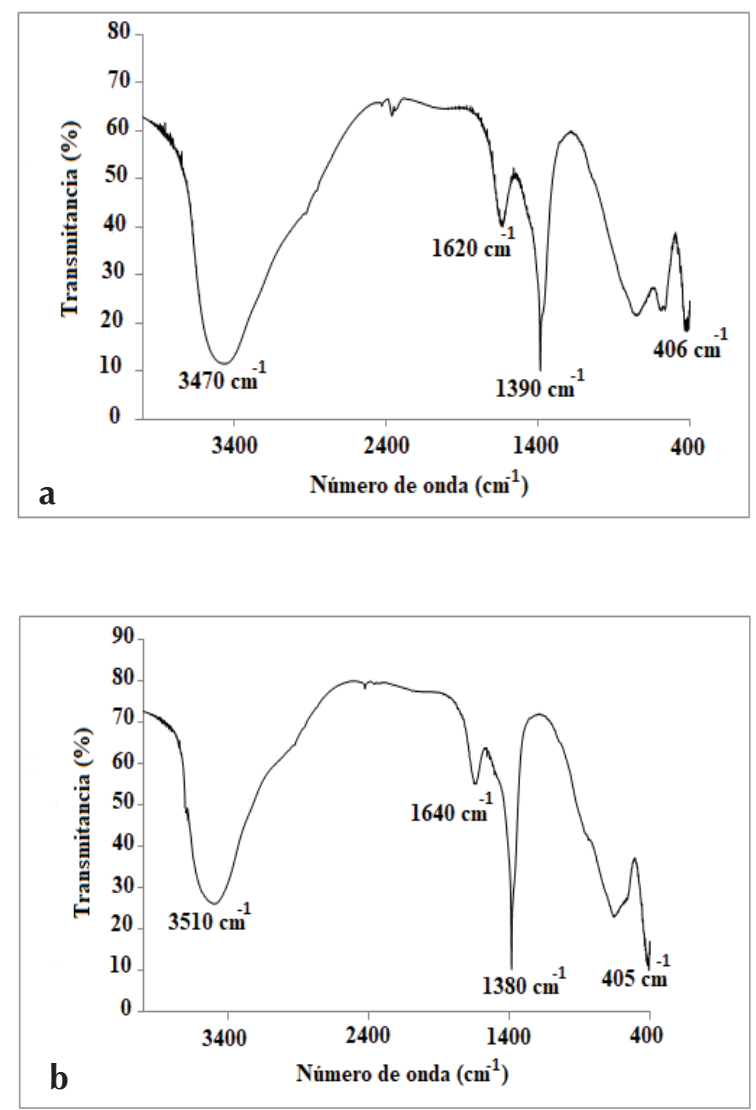

Figura 3. Espectro FT-IR de: a) la HT-Ni,

b) la HT-Mg 
El termograma, TGA, para las HTs sintetizada se presenta en la Figura 4.

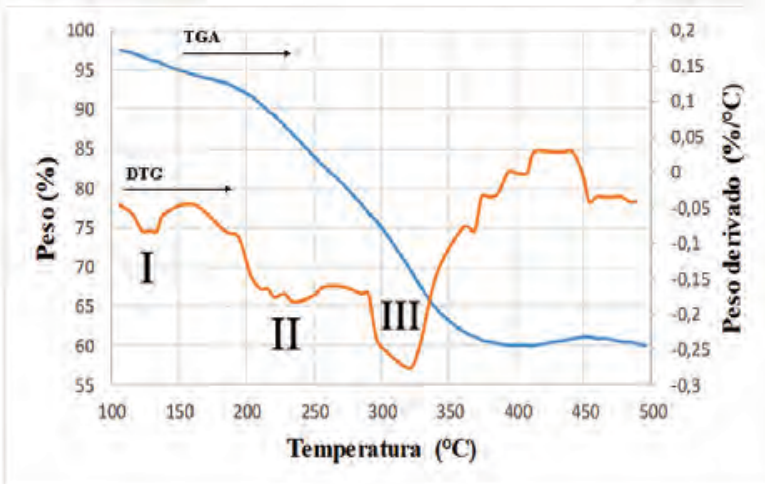

Figura 4. Termograma obtenido para las HTs sintetizadas (TGA-DTG)

La Figuras $5 a$ y 5b, muestran las curvas de calibración obtenidas mediante medidas de absorbancia de una serie de patrones estándar co- merciales de $\mathrm{Ni}, \mathrm{Mg}$ y Al y los residuales graficados en función de las coordenadas experimentales en el eje $x$, en las Figuras 6a-c. 

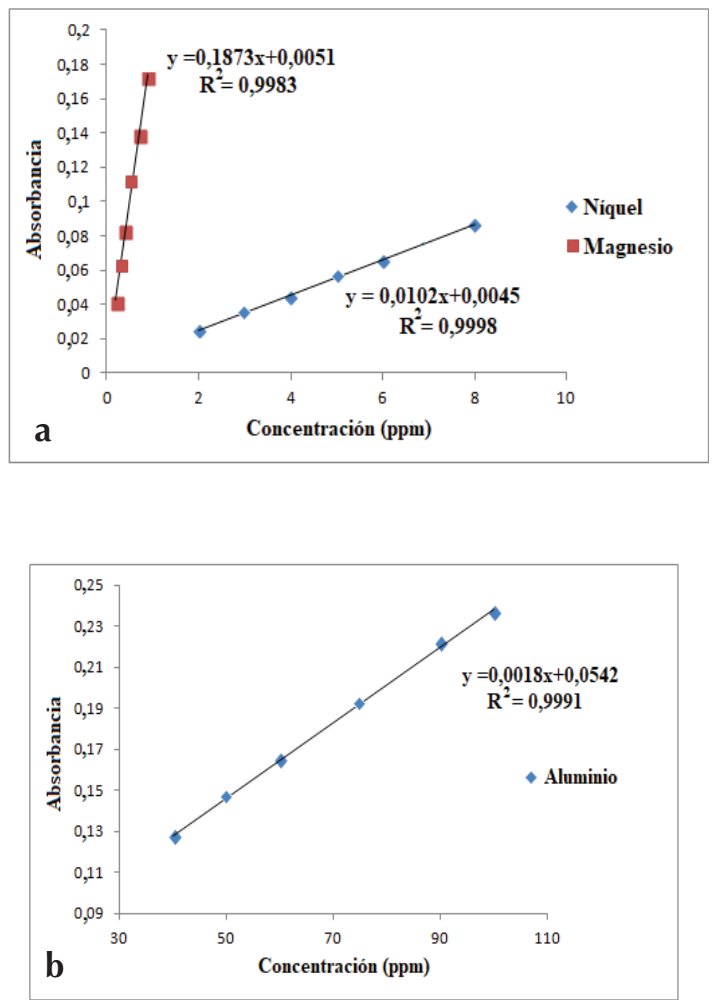

Figura 5. Curvas de calibración empleadas para la determinación de a) níquel y magnesio en las arcillas sintetizadas, b) aluminio en las arcillas sintetizadas 
InfoANALÍTICA 9(2)

Agosto 2021
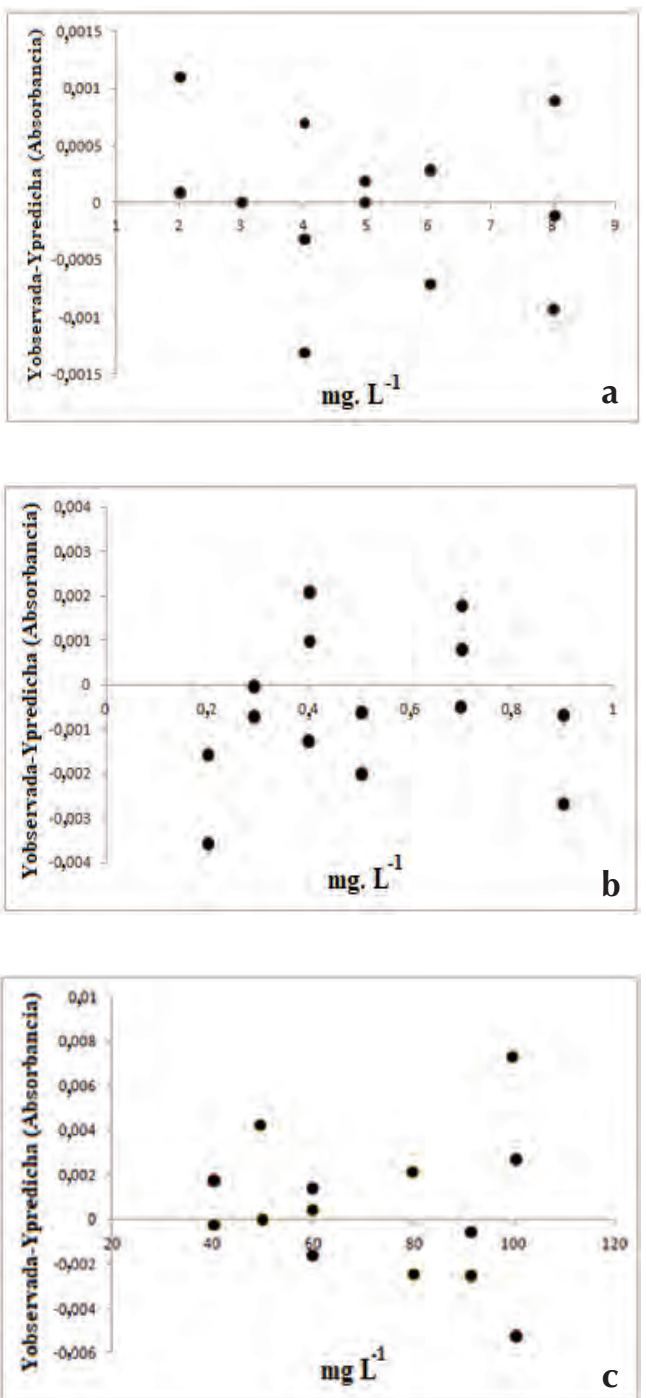

Figura 6. Residuales de la curva de calibración para la detección de: a) níquel, b) magnesio y, c) aluminio 
Las relaciones estequiométricas calculadas se muestran en la Tabla 1.

Tabla 1. Composición en metales de las HT sintetizadas, obtenidas por absorción atómica; fórmulas obtenidas para ambos materiales

\begin{tabular}{|c|c|c|c|}
\hline & $M g(\%)$ & $\mathrm{Al}(\%)$ & $\mathrm{Ni}(\%)$ \\
\hline $\mathrm{HT}-\mathrm{Mg}$ & \multicolumn{3}{|c|}{$(25,2 \pm 0,2) \quad(6,4 \pm 0,1)$} \\
\hline $\mathrm{HT}-\mathrm{Ni}$ & \multicolumn{2}{|c|}{$3,23 \pm 0,02) \quad(33,9 \pm 0,3)$} \\
\hline & \multicolumn{2}{|c|}{ Fórmulas obtenidas } \\
\hline $\mathrm{HT}-\mathrm{Mg}$ & $\mathrm{Mg}_{0,80} \mathrm{Al}_{0,20}(\mathrm{OH})_{2}\left(\mathrm{NO}_{3}\right)_{0,20}$ \\
\hline $\mathrm{HT}-\mathrm{Ni}$ & $\mathrm{Ni}_{0,82} \mathrm{Al}_{0,18}(\mathrm{OH})_{2}\left(\mathrm{NO}_{3}\right)_{0,18}$ \\
\hline
\end{tabular}

A modo comparativo frente a la técnica de espectroscopia de absorción atómica por Ilama, en la Tabla 2 se presenta la composición y fórmula para la HT-Ni obtenida por EDX (Figura 7).

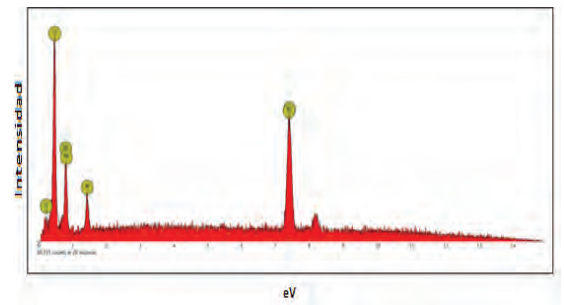

Figura 7. Análisis elemental por EDX de la HT-Ni
Tabla 2. Composición en metales de la HT-Ni sintetizada, obtenida por análisis elemental por EDX

\begin{tabular}{|c|}
\hline $\mathrm{Al}(\%) \quad \mathrm{Ni}(\%)$ \\
\hline$(33,5 \pm 0,1) \quad(33,5 \pm 0,1)$ \\
\hline Fórmula obtenida \\
\hline $\mathrm{Ni}_{0,81} \mathrm{Al}_{0,19}(\mathrm{OH})_{2}\left(\mathrm{NO}_{3}\right)_{0,19}$ \\
\hline
\end{tabular}

Las micrografías de ambas HTs, (Figura 8), muestran un material con partículas de un tamaño y formas variadas, con características típicas de los LHD.

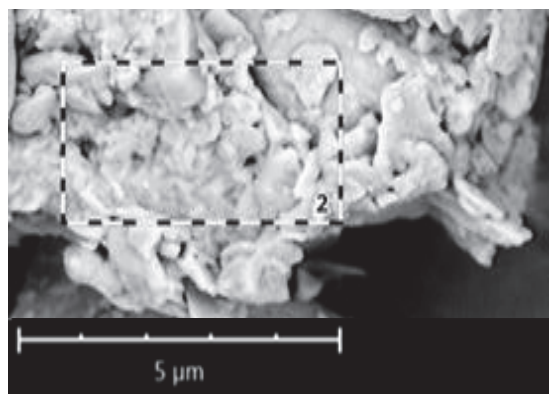

Figura 8. Micrografía, SEM, de las arcillas sintetizadas 


\section{DISCUSIÓN}

\section{Caracterización de las arcillas HTs modificada}

Los patrones de difracción de rayos $X$ para las HTs sintetizadas (Figuras 2a y 2 b), muestran patrones característicos de hidróxidos dobles laminares (Martínez \& Carvajal, 2012). Una serie de señales armónicas que se asignan a los planos (003), (006), (009). Para ambas arcillas, las señales (003) y (006) están ubicadas alrededor de 10 y 20 grados, $2 \theta$, mientras la señal del plano basal (003) se ubicó en $10,75^{\circ}$ para la HT-Ni y en $10,40^{\circ}$ para la HT-Mg. La señal más intensa, plano basal (003), se define como la distancia entre dos láminas contiguas análogas al plano (001), por lo que puede ser utilizada, mediante la ley de Bragg (Martínez \& Carvajal, 2012), para calcular la distancia entre los espacios interlaminares de las arcillas. Esta distancia fue de $8,23 \AA$ y $8,50 \AA$ A para la arcilla HT$\mathrm{Ni}$ y HT-Mg, respectivamente; separaciones comprendidas en el rango reportado para HTs que contienen aniones nitratos entre sus láminas (Miyata, 1983). Ambos patrones de difracción cuentan con una serie de señales asimétricas localizadas a partir de $35^{\circ}$, denotadas con los números (012) y (015), que representan los errores de apilamiento entre las láminas de brucita de las HTs (Fernández et al., 2006). El patrón de difracción para la HT-Mg presentó un pico de baja intensidad localizado en $60^{\circ} 2 \theta$ al que se le asignó el plano (110). Esta reflexión es equivalente a la distancia existente entre los cationes metálicos dentro de las láminas y al parámetro de celda a, con esta distancia es posible estimar el tamaño de los cristalitos mediante la ecuación de Scherrer (Martínez \& Carvajal, 2012), dando como resultado un tamaño de partícula de 38,92 nm y 8,44 nm para la HT-Mg y HT-Ni, respectivamente. Tomando en consideración, que para la determinación del tamaño de partícula mediante la ecuación de Scherrer, se realizó la aproximación a partículas de forma esféricas, y, conociendo la densidad promedio reportada para estos materiales de 2,06 g/ $\mathrm{cm}^{3}$; es posible generar un valor aproximado para las áreas superficiales de las hidrotalcitas sintetizadas, (Forano et al., 2013). Se obtuvieron áreas de $346 \mathrm{~m}^{2} / \mathrm{g}$ y 74,8 
$\mathrm{m}^{2} / \mathrm{g}$ para las HT-Ni y HT-Mg respectivamente; siendo este último valor $\left(74,8 \mathrm{~m}^{2} / \mathrm{g}\right)$, similar al reportado por otros trabajos con hidrotalcitas de $\mathrm{Mg}$ y $\mathrm{Al}$ de altas áreas superficiales (Zhang et al., 2019).

Dependiendo del grado de cristalinidad del material, es posible observar un mayor número de estas señales; en este caso la HT-Mg presenta un mayor grado de cristalinidad, lo que se evidencia por picos en el difractograma mucho más delgados y definidos que los obtenidos para la HT-Ni.

Los espectros IR obtenidos para los materiales, muestran bandas de transmitancia reportadas en anterioridad para las arcillas tipo HTs (Wiyantoko et al., 2015) (Figura 3a y 3b). En ambas HTs se observan bandas amplias e intensas, $3470 \mathrm{~cm}^{-1}$ para la HT-Ni y $3510 \mathrm{~cm}^{-1}$ para la HT-Mg, asociadas a enlaces $\mathrm{O}-\mathrm{H}$ del hidróxido doble, la señal intensa en la región cercana a $1390 \mathrm{~cm}^{-1}$ a la vibración de los enlaces $\mathrm{N}=\mathrm{O}$ de los nitratos contenidos en el espacio interlaminar (Fernández et al., 2006), la señal débil entre 406 y $405 \mathrm{~cm}^{-1}$ a la vibración de los enlaces O-M-O y el conjunto de señales anchas entre 550 y $800 \mathrm{~cm}^{-1}$ es atribuido a los enlaces $\mathrm{M}-\mathrm{O}$.

El termograma, TGA y DTG, para las HTs sintetizada se presenta en la Figura 4 . La pérdida de peso (15\%) alrededor de $100{ }^{\circ} \mathrm{C}$ (I) es característico de la eliminación del $\mathrm{H}_{2} \mathrm{O}$ fisiosorbido y el $\mathrm{H}_{2} \mathrm{O}$ entre capas de las $\mathrm{HT}$. La segunda pérdida (30\%) entre 200 y $250{ }^{\circ} \mathrm{C}$ (II) se atribuye al detrimento de una mayor cantidad de agua interlaminar junto a la eliminación de especies carbonatos que pudieron ser retenidas en el espacio interlaminar durante el proceso de síntesis. La última pérdida, a partir de los $300{ }^{\circ} \mathrm{C}$ (III) está relacionada con la deshidroxilación, descarbonatación y formación de óxido metálicos mixtos amorfos (Edañol et al., 2020; Ferreira et al., 2004; Yaseneva et al., 2018).

Las Figuras $5 a$ y 5b, muestran las curvas de calibración obtenidas mediante medidas de absorbancia de una serie de patrones estándar comerciales de Ni, Mg y Al. Las medidas se realizaron después de disolver muestras de estas en ácido nítrico diluido $\left(0,1 \mathrm{~mol} \mathrm{~L}^{-1}\right)$. Las tres curvas de calibración muestran coeficientes de correlación aceptables en el ajuste, 
con $R^{2}=0,9998,0,9991$ y 0,9983 para $\mathrm{Ni}, \mathrm{Mg}$ y $\mathrm{Al}$, respectivamente. No obstante, el valor de $\mathrm{R}^{2}$ no representa un criterio definitivo para evaluar la validez de una regresión lineal, por lo cual se realizó la representación de los residuales (Rodríguez et al., 1996). Los residuales fueron graficados en función de las coordenadas experimentales en el eje $x$, lo que corresponde a unidades de $\mathrm{mg} \mathrm{L}^{-1}$, (Figuras 6a-c). Se obtuvo una distribución aleatoria de los puntos calculados con una falta de dependencia entre las magnitudes en los ejes " $x$ " $y$ " $y$ ", siendo esto evidencia de un buen ajuste que cuenta sólo con los errores aleatorios introducidos por la labor del experimentador (Rawski et al., 2016). La regresión lineal de las curvas de calibración arroja valores para los puntos de corte de $(0,0045 \pm 0,0060)$, $(0,0051 \pm 0,0070)$ y $(0,0542 \pm$ 0,0300) para el Ni, Mg y el Al, respectivamente. En la determinación de los primeros dos elementos, el punto de corte contiene al origen al tomar en cuenta la dispersión que acompaña a "b", este fenómeno corresponde con lo esperado teóricamente, ya que, una concentración teórica de 0 del elemento debería producir una absorbancia nula si se eliminan los posibles efectos de las matrices.

La curva obtenida para la determinación del Al no posee un punto de corte nulo, efecto que atribuimos al cambio de las condiciones de trabajo, ya que en los dos primeros metales los gases utilizados para la llama del espectrofotómetro son una combinación de aire-acetileno, y en el caso del Al, se utilizó aire-acetileno-óxido nitroso. La Tabla 1, resume las composiciones encontradas de los metales junto con la formula química derivada de estas magnitudes. Las relaciones estequiométricas mostradas en la tabla, se aproximan a las reportadas en la literatura $\mathrm{Mg}_{0,75} \mathrm{Al}_{0,25}(\mathrm{OH})_{2}\left(\mathrm{NO}_{3}\right)_{0,25}$ para la HT-Mg y $\mathrm{Ni}_{0,75} \mathrm{Al}_{0,25}(\mathrm{OH})_{2}\left(\mathrm{NO}_{3}\right)_{0,25}$ para la HT-Ni (Misra \& Perrotta, 1992); la pequeña diferencia puede deberse a la naturaleza no estequiométrica característica de las HT, que pueden producir pequeñas cantidades de óxidos dobles amorfos frente a temperaturas elevadas en el proceso de secado, lo que lleva a obtener materiales de composiciones, hasta cierto grado, no homogéneas (Balsamo et al., 2012). Sin embargo, 
dicho fenómeno no limita en gran medida la capacidad de intercambio iónico de las arcillas HTs, una de las principales propiedades de estos materiales, debido a que los hidróxidos dobles laminares con buenos grados de cristalinidad se han reportado con composiciones muy variadas en donde el índice del metal trivalente puede encontrarse entre 0,15 y 0,40 (Kannan et al., 1995).

Con el fin de descartar que las diferencias observadas en las composiciones teóricas y las experimentales se deban a algún error cometido en la determinación del contenido de metales mediante espectroscopia de absorción atómica, se determinó la composición de metales en las arciIlas mediante análisis elemental por EDX, Tabla 2. Los resultados obtenidos para la composición de la HT-Ni no muestran diferencias significativas con los valores encontrados mediante absorción atómica, por lo que se concluye que las fórmulas derivadas de las medidas por esta última técnica representan una buena aproximación de la composición de las arcillas sintetizadas, y no son resultado de un error en la ejecución experimental. Por otro lado, el análisis elemental, Figura 7, indicó la presencia de pequeñas cantidades de carbono en la arcilla. El carbono se detecta de la porción de aniones carbonatos que inevitablemente se intercalan en espacio interlaminar durante el lavado y el secado, respuesta de la alta afinidad que presentan estas arcillas frente al anión $\mathrm{CO}_{3}{ }^{2-}$ a la hora de intercambiar iones (lyi et al., 2004), cuyo confinamiento puede ayudar a la disminución del tamaño de partícula (Solovov et al., 2018).

A pesar de que la morfología de los hidróxidos doble laminares se encuentra estrechamente vinculada a las condiciones de síntesis, las cuales, pueden variar de forma considerable entre las referencias consultadas, existen algunas características en común que comparten estos materiales y que pueden ser utilizadas con el fin de caracterizarlos e identificaros como compuestos tipo HT.

Las micrografías de ambas HTs, Figura 8 , muestran un material con partículas de un tamaño y formas variadas. No obstante, los granos más pequeños poseen dimensiones inferiores a 5 micras y una morfología tipo hojuelas con bordes afilados 
propensas a la exfoliación (centro de la foto de la derecha), lo cual es característico de los hidróxidos dobles laminares sintéticos (Cocheci et al., 2010).

\section{CONCLUSIÓN}

Mediante el método de precipitación acoplado a una titulación por precipitación doble, se sintetizaron HTs con la composición química $\mathrm{Ni}_{0,82} \mathrm{Al}_{0,18}(\mathrm{OH})_{2}\left(\mathrm{NO}_{3}\right)_{0,18}$ $\mathrm{Mg}_{0,80} \mathrm{Al}_{0,20}(\mathrm{OH})_{2}\left(\mathrm{NO}_{3}\right)_{0,20}$.

Determinaciones por espectroscopia de absorción atómica y análisis elemental por EDX permitieron corroborar las fórmulas propuestas. Los patrones de difracción de rayos $X$ presentaron una serie de picos típicos de materiales tipo LDH y mediante la ecuación de Scherrer se obtuvo que las HTs sintetizadas poseen tamaño nanométrico; la aproximación de las partículas de las arcillas a formas esféricas permitió estimar áreas específicas elevadas de 74,8 m²/g y 346 $\mathrm{m}^{2} / \mathrm{g}$ para las HT-Mg y HT-Ni respectivamente. Espectros de FTIR de los materiales indicaron la presencia de las señales esperadas para los materiales sintetizados y las caracterizaciones por SEM indicaron partículas con la morfología típica de plaquetas de LDH.

\section{AGRADECIMIENTOS}

Los autores agradecen el apoyo de la Pontificia Universidad Católica del Ecuador, Universidad Simón Bolívar Caracas-Venezuela y la Corporación Ecuatoriana para el Desarrollo de la Investigación y la Academia (CEDIA).
Dedicamos este trabajo a José Domínguez (1946-2020), a quien recordaremos siempre por su incansable trabajo y amor a la ciencia. 


\section{LISTA DE REFERENCIAS}

Bahramian, A. (2020). The effect of thermal and non-thermal routes on treatment of the Mg-Al layered double hydroxide catalyst dispersed by titania nanoparticles in products distribution arising from poly (ethylene terephthalate) degradation. Polymer Degradation and Stability, 179, 109243. https://doi.org/10.1016/j.polymdegradstab.2020.109243

Balsamo, N., Mendieta, S., Oliva, M., Eimer, G., Crivello, M. (2012). Synthesis and characterization of metal mixed oxides from Layered Double Hydroxides. Procedia Materials Science, 1, 506-513. https://doi.org/10.1016/j.mspro.2012.06.068

Britto, S., Kamath, P.V. (2014). Synthesis, structure refinement and chromate sorption characteristics of an Al-rich bayerite-based layered double hydroxide. Journal of Solid State Chemistry, 215, 206-210. https://doi.org/10.1016/j.jssc.2014.02.031

Chaillot, D., Bennici, S., Brendlé, J. (2020). Layered double hydroxides and LDH-derived materials in chosen environmental applications: a review. Environmental Science and Pollution Research International, 28, 24375-24405. https://doi.org/10.1007 /s11356-020-08498-6

Cocheci, L., Barvinschi, P., Pode, R., Popovici, E., Seftel, E.M. (2010). Structural Characterization of Some Mg/Zn-Al Type Hydrotalcites Prepared for Chromate Sorption from Wastewater. Chemical Bulletin of "Politehnica" University of Timisoara, ROMANIA Series of Chemistry and Environmental Engineering, 55(69), 40-45.

Dahdah, E., Estephane, J., Haydar, R., Youssef, Y., El Khoury, B., Gennequin, C., Aboukaïs, A., Abi-Aad, E., Aouad. S. (2020). Biodiesel production from refined sunflower oil over Ca-Mg-Al catalysts: Effect of the composition and the thermal treatment. Renewable Energy, 146, 1242-1248. https://doi.org/10.1016/j.renene.2019.06.171

Edañol, Y.D.G., Poblador, J.A.O., Talusan, T. J. E., Payawan L. M. (2020). Co-precipitation synthesis of Mg-Al-CO3 layered double hydroxides and its adsorption kinetics with phosphate (V) ions. Materials Today: Proceedings, 33(4), 1809-1813. https://doi. org/10.1016/j.matpr.2020.05.059 
Fernández, L., Borrás, C., Carrero, H. (2006). Electrochemical behavior of phenol in alkaline media at hydrotalcite-like clay/ anionic surfactants/glassy carbon modified electrode. Electrochimica Acta, 52, 872-884. https://doi.org/10.1016/j.electacta .2006 .06 .021

Ferreira, O.P., Alves, O. L., Gouveia, D. X., Souza Filho, A.G., de Paiva, J. A., Mendes Filho, J. (2004). Thermal decomposition and structural reconstruction effect on Mg-Fe-based hydrotalcite compounds. Journal of Solid State Chemistry, 177(9), 3058-3069. https://doi.org/10.1016/j.jssc.2004.04.030

Forano, C., Costantino, U., Prévot, V., Gueho, C.T. (2013). Layered Double Hydroxides (LDH). Developments in Clay Science, 5, 745-782. https://doi.org/10.1016/B9780-08-098258-8.00025-0

Gevers, B.R., Naseem, S., Leuteritz, A., Labuschagné, F.J. (2019). Comparison of nanostructured transition metal modified tri-metal MgMAI-LDHs (M= Fe, Zn, Cu, Ni, Co) prepared using co-precipitation. RSC advances, 9(48), 28262-28275. https://doi.org/10.1039/C9RA05452A

Hawthorne, F.C., Cooper M.A. (2013). The crystal structure of chalcoalumite: mechanisms of Jahn-Teller-driven distortion in ${ }^{[6]} \mathrm{Cu}^{2+}$-containing oxysalts. Mineralogical Magazine, 77(7), 2901-2912. https://doi.org/10.1180/minmag.2013.077.7.02

Hu, Y., Pan, C., Zheng, X., Liu, S., Peng, X. (2021). Synthesis of NiSiO@ NiAlFe by Hydrothermal method and removal of $\mathrm{Cs}+$ in water. IOP Conference Series: Earth and Environmental Science, 631(1), 012008. http://dx.doi.org/10.1088/17551315/631/1/012008

Iyi, N., Matsumoto, T., Kaneko, Y., Kitamura, K. (2004). Deintercalation of Carbonate Ions from a Hydrotalcite-Like Compound: Enhanced Decarbonation Using AcidSalt Mixed Solution. Chemistry of Materials, 16(15), 2926-2932. https://doi.org/10. 1021/cm049579g

Jeung, D.G., Kim, T.H., Oh, J.M. (2020). Homogeneous Incorporation of Gallium into Layered Double Hydroxide Lattice for Potential Radiodiagnostics: Proof-of-Concept. Nanomaterials, 11(1), 44. 
Kannan, S., Rives, V., Knözinger, H. (2004). High-temperature transformations of Curich hydrotalcites. Journal of Solid State Chemistry, 177(1), 319-331. https://doi.org /10.3390/nano11010044

Kannan, S., Velu, S., Ramkumar, V., Swamy, C.S. (1995). Synthesis and physicochemical properties of cobalt aluminium hydrotalcites. Journal of Materials Science, 30, 1462-1468. https://doi.org/10.1007/BF00375249

Li, S., Shi, Y., Yang, Y., Zheng, Y., Cai, N. (2013). High-performance CO2 adsorbent from interlayer potassium-promoted stearate-pillared hydrotalcite precursors. Energy \& Fuels, 27(9), 5352-5358. https://doi.org/10.1021/ef400914r

Li, W., Jiang, Y., Yang, M., Qu, M., Li, Y., Shen, W., He, R., Li, M. (2021). Controlled synthesis of hierarchical hollow CoLDH nanocages electrocatalysts for oxygen evolution reaction. Chemical Physics, 541, 111011. https://doi.org/10.1016/j .chemphys.2020.111011

Liu, B., Zhang, M., Wang, Y., Chen, Z., Yan, K. (2021). Facile synthesis of defect-rich ultrathin NiCo-LDHs, NiMn-LDHs and NiCoMn-LDHs nanosheets on Ni foam for enhanced oxygen evolution reaction performance. Journal of Alloys and Compounds, 852, 156949. https://doi.org/10.1016/j.jallcom.2020.156949

Manivannan, R., Karthikeyan, C. (2013). Synthesis of Biodiesel from Neem Oil Using Mg-Al Nano Hydrotalcite. Advanced Materials Research, 678, 268-272. https://doi.org/10.4028/www.scientific.net/AMR.678.268

Martínez, D., Carvajal, G. (2012). Hidróxidos Dobles laminares: arcillas sintéticas con aplicaciones en nanotecnología. Avances en Química, 7(1), 87-99.

Mishra, G., Dash, B., Pandey, S. (2018). Layered double hydroxides: A brief review from fundamentals to application as evolving biomaterials. Applied Clay Science, 153, 172-186. https://doi.org/10.1016/j.clay.2017.12.021

Misra, C., Perrotta, A.J. (1992). COMPOSITION AND PROPERTIES OF SYNTHETIC HYDROTALCITES. Clays and Clay Minerals. 40(2), 145-150. https://doi.org/10. 1346/CCMN.1992.0400202 
Miyata, S. (1983). Anion- Exchange Properties of Hydrotalcite Like Compounds. Clays and Clay minerals, 31(4), 305-311. https://doi.org/10.1346/CCMN.1983.0310409

Pan, S., Li, B., Yu, J., Zhao, L., Zhang, Y. (2021). Composition controllable fabrication of ultrathin 2D CoMn layered double hydroxides for highly efficient electrocatalytic oxygen evolution. Applied Surface Science, 539, 148305. https://doi.org/10. 1016/j.apsusc.2020.148305

Pérez, A.G., Paredes-Carrera, S.P., Martínez-Gutiérrez, H., Sánchez-Ochoa, J.C., PérezGutiérrez, R.M., Cayetano-Castro, N. (2020). Effect of combined microwave-ultrasound irradiation in the structure and morphology of hidrotalcite like compounds $\mathrm{Al} / \mathrm{Mg}-\mathrm{CH}_{3} \mathrm{COO}$ and its evaluation in the sorption of a reactive dye. Revista Mexicana de Ingeniería Química, 19(1), 363-375. https://doi.org/10.24275 /rmiq/Mat567

Pizzoferrato, R., Richetta, M. (2020). Layered Double Hydroxides (LDHs). Crystals, 10(12), 1121.

Rawski, R.I., Sanecki, P.T., Kijowska, K.M., Skital, P. M., Saletnik, D.E. (2016). Regression Analysis in Analytical Chemistry. Determination and Validation of Linear and Quadratic Regression Dependencies. South African Journal of Chemistry, 69,166-173. http://dx.doi.org/10.17159/0379-4350/2016/v69a20

Rodríguez, L.C., Campana, A.M.G., Sendra, J.M.B. (1996). Statistical estimation of linear calibration range. Analytical Letters, 29(7), 1231-1239. https://doi.org/10.1080 /00032719608001471

Rybka, K., Matusik, J., Kuligiewicz, A., Leiviskä, T., Cempura, G. (2021). Surface chemistry and structure evaluation of $\mathrm{Mg} / \mathrm{Al}$ and $\mathrm{Mg} / \mathrm{Fe} \mathrm{LDH}$ derived from magnesite and dolomite in comparison to LDH obtained from chemicals. Applied Surface Science, 538, 147923. https://doi.org/10.1016/j.apsusc.2020.147923

Shekoohi, K., Hosseini, F.S., Haghighi, A. H., Sahrayian, A. (2017). Synthesis of some Mg/Co-Al type nano hydrotalcites and characterization. MethodsX, 4, 86-94. https://doi.org/10.1016/j.mex.2017.01.003 
Solovov, V.A., Nikolenko, N.V., Kovalenko, V.L., Kotok, V.A., Burkov, A.A., Kondrat'ev, D.A., Chernova, O.V., Zhukovin, S.V. (2018). Synthesis of Ni (II)-Ti (IV) layered double hydroxides using coprecipitation at high supersaturation method. ARPN Journal of Engineering and Applied Sciences, 13(24), 9652-9656.

Stamate, A.E., Pavel, O.D., Zavoianu, R., Marcu, I.C. (2020). Highlights on the Catalytic Properties of Polyoxometalate-Intercalated Layered Double Hydroxides: A Review. Catalysts, 10(1), 57. https://doi.org/10.3390/catal10010057

Wen, N., Su, Y., Deng, W., Zhou, H., Zhao, B. (2021). Selective catalytic reduction of $\mathrm{NO}$ with $\mathrm{C} 3 \mathrm{H} 6$ over CuFe-containing catalysts derived from layered double hydroxides. Fuel, 283, 119296. https://doi.org/10.1016/j.fuel.2020.119296

Wiyantoko, B., Kurniawati, P., Purbaningtias, T.E., Fatimah, I. (2015). Synthesis and Characterization of Hydrotalcite at Different Mg/AI Molar Ratios. Procedia Chemistry, 17, 21 - 26. https://doi.org/10.1016/j.proche.2015.12.115

Wu, L., Peng, B., Li, Q., Wang, Q., Yan, X., Lin, Q., Ji, C. (2019). Formation of high crystaIline LDH sludge for removing $\mathrm{Cu}$ and $\mathrm{Zn}$ from wastewater by controlled doublejet precipitation. Environmental Science and Pollution Research, 26(19), 19665-19675. https://doi.org/10.1007/s11356-019-05161-7

Yao, J., Huang, R., Jiang, J., Xiao, S., Li, Y. (2021). Lithium storage performance of $\alpha-\mathrm{Ni}$ $(\mathrm{OH}) 2$ regulated by partial interlayer anion exchange. Ionics, 27, 1125-1135. https://doi.org/10.1007/s11581-020-03889-8

Yaseneva, P., An, N., Finn, M., Tidemann, N., Jose, N., Voutchkova-Kostal, A., Lapkin, A. (2019). Continuous synthesis of doped layered double hydroxides in a meso-scaleflow reactor. Chemical Engineering Journal, 360, 190-199.

Zaghloul, A., Benhiti, R., Abali, M.H., Ichou, A.A., Soudani, A., Chiban, M., Zerbet. M., Sinan, F. (2021). Kinetic, isotherm, and thermodynamic studies of the removal of methyl orange by synthetic clays prepared using urea or coprecipitation. Euro-Mediterranean Journal for Environmental Integration, 6(1), 1-10. https://doi.org/10 .1007/s41207-020-00217-4 
Zhang, Z., Chen, S., Zhang, Y., (2019). Effect of hydrotalcite-like compounds with high specific surface area on mechanical properties and carbonation resistance of cementitious composites. Material Research. Express, 6, 115099. https://doi.org/10 $.1088 / 2053-1591 / a b 4 b 89$

Zheng, N., Yu, Y., Shi, W., Yao, H. (2019). Biochar suppresses N 2 O emissions and alters microbial communities in an acidic tea soil. Environmental Science and Pollution Research, 26(35), 35978-35987. https://doi.org/10.1007/s11356-019-06704-8

Zhitova, E.S., Greenwell, H.C., Krzhizhanovskaya, M.G., Apperley, D.C., Pekov, I.V., Yakovenchuk, V.N. (2020). Thermal Evolution of Natural Layered Double Hydroxides: Insight from Quintinite, Hydrotalcite, Stichtite, and lowaite as Reference Samples for CO3-and Cl-Members of the Hydrotalcite Supergroup. Minerals, 10(11), 961. https://doi.org/10.3390/min10110961 